\title{
Improved Riccati transfer matrix method for free vibration of non-cylindrical helical springs including warping
}

\author{
A.M. Yu* and Y. Hao \\ School of Aerospace Engineering and Applied Mechanics, Tongji University, Shanghai, China \\ Received 1 November 2011 \\ Revised 13 January 2012
}

\begin{abstract}
Free vibration equations for non-cylindrical (conical, barrel, and hyperboloidal types) helical springs with noncircular cross-sections, which consist of 14 first-order ordinary differential equations with variable coefficients, are theoretically derived using spatially curved beam theory. In the formulation, the warping effect upon natural frequencies and vibrating mode shapes is first studied in addition to including the rotary inertia, the shear and axial deformation influences. The natural frequencies of the springs are determined by the use of improved Riccati transfer matrix method. The element transfer matrix used in the solution is calculated using the Scaling and Squaring method and Pad'e approximations. Three examples are presented for three types of springs with different cross-sectional shapes under clamped-clamped boundary condition. The accuracy of the proposed method has been compared with the FEM results using three-dimensional solid elements (Solid 45) in ANSYS code. Numerical results reveal that the warping effect is more pronounced in the case of non-cylindrical helical springs than that of cylindrical helical springs, which should be taken into consideration in the free vibration analysis of such springs.
\end{abstract}

Keywords: Non-cylindrical helical spring, warping effect, improved Riccati transfer matrix method, natural frequency, mode shape

\section{Introduction}

Vehicle suspension system plays an important role in improving ride comfort and manipulation stability of the vehicle, and decreasing the damage of parts caused by dynamic load. To make arrangement of the vehicle suspension system more compact, the variable-stiffness coil springs have been used in many parts of machines, especially in automobiles. Therefore, the free vibration problem of non-cylindrical helical springs with variable centerline radius and step for unit angle of the helix is of great importance from an engineering point of view.

Epstein [1] theoretically derived both the elongations and fundamental frequencies of conical helical springs for several dynamic boundary conditions and experimentally verified the frequencies on nickel and piano wire springs. After his pioneering work, the vibration behavior of non-cylindrical helical springs has been the subject of research in the last three decades. Nagaya et al. [2] studied the free vibration analysis for barrel and hyperboloidal types of helical springs numerically and experimentally. They used the static element transfer matrix that was deduced in closed form taking into consideration only the axial deformations. Yildirim [3] used both the Myklestad method and the complementary functions methods, and presented the free vibration of non-cylindrical helical springs taking into consideration the effects of axial and shear deformations together with the rotary inertia. Yildirim et al. [4,5]

*Corresponding author: A.M. Yu, School of Aerospace Engineering and Applied Mechanics, Tongji University, Shanghai, China. Tel.: +86 21 65983267; Fax: +86 21 65983267; E-mail: aimin.yu@163.com. 
and Yildirim [6] investigated free vibration of unidirectional composite non-cylindrical helical springs with the help of the transfer matrix method. The rotary inertia, axial and shear deformation effects were taken into account in the solution based on the first-order shear deformation theory. Yildirim and Ince [7] have presented the equations of motion of non-cylindrical helical springs in Frenet coordinates. In this work, all the derivatives were with respect to the arc length coordinate along the beam. Wu and Hsu [8] developed an analytical model for a particular type of conical spring. Their study focuses on a conical spring with a constant helix angle that does not telescope, and with cylindrical closed and ground ends. This model gives spring deflection as a function of load. Recently, Busool and Eisenberger [9] attained the exact shape functions having terms less than 200 to derive the dynamic stiffness matrix of arbitrary shaped helices with variable cross-section by considering the effects of axial, shear deformations and the rotary inertia, which used the differential equations of motion for the springs developed in Ref. [7].

The effects of vibrational parameters on the natural frequencies of non-cylindrical helical springs have been investigated in most of the work mentioned above, but the shapes of wire cross-section for these springs are mostly circular, only relatively few studies on the free vibration of such springs with square cross-section can be found [3,9, 10]. It is worth notice that for the springs with square cross-section, whether they are cylindrical or non-cylindrical helical springs, there is no investigation published on the vibration analysis of the springs taking into consideration the warping effect upon the natural frequencies. Calım [10] pointed out that the warping effect in the vibration analysis of the springs should be considered. Recently Yu et al. [11] worked out the effect of warping behavior in the formulation on the free vibration characteristics of cylindrical helical springs with noncircular cross-sections, and calculations show that even for cylindrical helical springs, the warping effect upon natural frequencies is also evident.

The differential equations of motion for non-cylindrical helical springs in terms of displacements, internal forces, generalized warping coordinate and generalized warping moment, based on spatially curved beam theory, can be derived. They consist of 14 first-order partial differential equations with variable coefficients. The resulting system of equations will become a "stiff-conditioning" system of equations due to increase of two degrees of freedom containing the generalized warping coordinate and the generalized warping moment, therefore, the traditional transfer matrix method is no longer suitable for solving the boundary value problems of stiff ordinary differential equations. In recent years, some new methods for multibody dynamics, repetitive structures, elastic beams, multirotor systems and nonlinear rotor-bearing systems, Riccati discrete time transfer matrix method, improved Riccati transfer matrix method, whole transfer matrix method and CSDT-Riccati transfer matrix integration method have been proposed [12-17]. In this paper, the scalar free vibration equations obtained by assuming harmonic motion are solved numerically using improved Riccati transfer matrix method [18,19], and the element transfer matrix used in the solution is calculated using the Scaling and Squaring method and Pad'e approximations [20]. After verifying the results obtained in this study and given by a finite element model, a discussion about the effects of vibrational parameters on the natural frequencies has also been presented.

\section{Geometry and formulations of non-cylindrical helical springs}

Assuming that the centroid of wire cross-section coincides with its shear center, both normal and binormal axes are the centroid principal axes, and that the rods made of an linear elastic, homogenous and isotropic material are considered by including the warping effect due to torsion.

The parametric equation of a non-cylindrical helix in Fig. 1 is given:

$$
\begin{aligned}
& x=R(\beta) \cos \beta, \quad y=R(\beta) \sin \beta, \quad z=h(\beta) \beta \\
& h(\beta)=R(\beta) \tan \bar{\alpha}
\end{aligned}
$$

where $\bar{\alpha}$ denotes the pitch angle, while $R(\beta)$ and $h(\beta)$ denote the centerline radius and the step for unit angle of the helix as a function of the horizontal angle $\beta$, respectively. In the cartesian coordinate system, as seen in Fig. 1, the position vector any point on the helix can be expressed as $\mathbf{r}=x \mathbf{i}+y \mathbf{j}+z \mathbf{k}$. While in the Frenet coordinate system, the Frenet unit vectors, associated with the bar-axis, are expressed as 


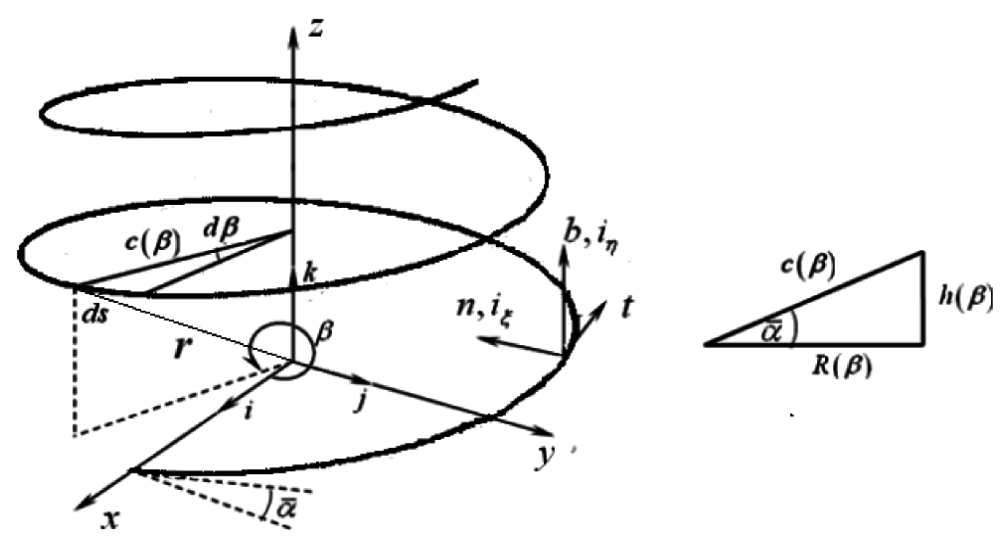

Fig. 1. Geometry of a typical non-cylindrical helix.

$$
\mathbf{t}=\frac{d \mathbf{r}}{d s}, \quad \mathbf{i}_{\xi}=\frac{d \mathbf{t} / d s}{d t / d s}, \quad \mathbf{i}_{\eta}=\mathbf{t} \times \mathbf{i}_{\xi}
$$

where $\mathbf{t}, \mathbf{i}_{\xi}, \mathbf{i}_{\eta}$ are the tangential, normal and binormal unit vectors, respectively, and the infinitesimal arc length of the helix is defined as

$$
d s=\sqrt{d x^{2}+d y^{2}+d z^{2}}=\sqrt{R^{2}(\beta)+h^{2}(\beta)} d \beta=c(\beta) d \beta
$$

The following differential relations among the unit vectors $\mathbf{t}, \mathbf{i}_{\xi}, \mathbf{i}_{\eta}$ can be obtained with the aid of the Frenet formulas [21]

$$
\frac{d \mathbf{t}}{d s}=k_{\eta} \mathbf{i}_{\xi}, \quad \frac{d \mathbf{i}_{\xi}}{d s}=-k_{\eta} \mathbf{t}+k_{s} \mathbf{i}_{\eta}, \quad \frac{d \mathbf{i}_{\eta}}{d s}=-k_{s} \mathbf{i}_{\xi}
$$

where $k_{\eta}=R(\beta) / c^{2}(\beta)$ and $k_{s}=h(\beta) / c^{2}(\beta)$ are the curvature and torsion of the helix axis, respectively. Figure 2 describes the different types of helical springs with four cross- sectional shapes. The centerline radius of any point on the helix axis for both barrel and hyperboloidal springs can be determined by

$$
R(\beta)=R_{1}+\left(R_{2}-R_{1}\right)\left(1-\frac{\beta}{\pi n}\right)^{2}
$$

and for conical spring

$$
R(\beta)=R_{1}+\frac{\left(R_{2}-R_{1}\right) \beta}{2 \pi n}
$$

where $(0 \leqslant \beta \leqslant 2 \pi n)$ and $n$ is the number of active turns of the helix.

Now, the spatially curved system is taken as a special case of a non-cylindrical helical spring, and the governing differential equations of motion for the spring, using naturally curved and twisted beam theory [22], can be obtained by simple inserting $k_{\xi}=0$ and the external force and moments per unit length on the axis of the spring $\mathbf{p}(s, t)=\mathbf{m}(s, t)=0$. Thus,

$$
\begin{aligned}
& m \ddot{u}_{\xi}=Q_{\xi}^{\prime}-k_{s} Q_{\eta}+k_{\eta} Q_{s} \\
& m \ddot{u}_{\eta}=Q_{\eta}^{\prime}+k_{s} Q_{\xi} \\
& m \ddot{u}_{s}+\iint \rho \chi d \xi d \eta \ddot{\alpha}=Q_{s}^{\prime}-k_{\eta} Q_{\xi} \\
& I_{m \xi} \ddot{\varphi}_{\xi}+\iint \rho \eta \chi d \xi d \eta \ddot{\alpha}=M_{\xi}^{\prime}-k_{s} M_{\eta}+k_{\eta} M_{s}-Q_{\eta}
\end{aligned}
$$




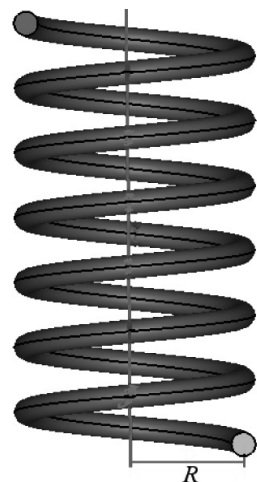

(a)

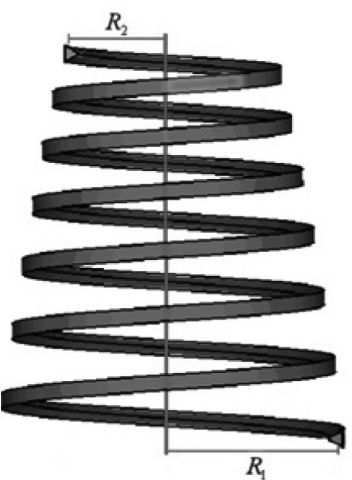

(b)

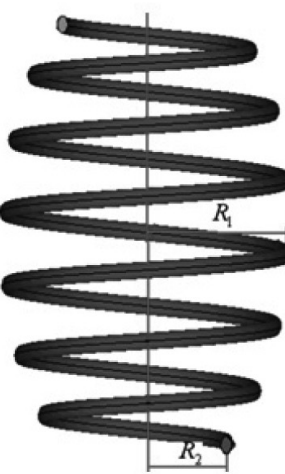

(c)

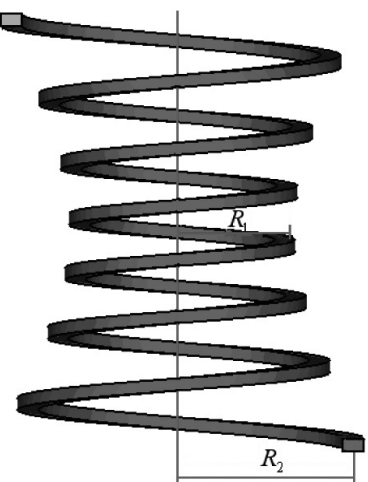

(d)

Fig. 2. The different types of helical springs with four cross-sectional shapes: (a) $R_{\max }=R(\mathrm{~b}) R_{\max }=R_{1}(\mathrm{c}) R_{\max }=R_{1}(\mathrm{~d}) R_{\max }=R_{2}$.

$$
\begin{aligned}
& I_{m \eta} \ddot{\varphi}_{\eta}-\iint \rho \xi \chi d \xi d \eta \ddot{\alpha}=M_{\eta}^{\prime}+k_{s} M_{\xi}+Q_{\xi} \\
& I_{m p} \ddot{\varphi}_{s}=M_{s}^{\prime}-k_{\eta} M_{\xi} \\
& \rho \iint \chi^{2} d \xi d \eta \ddot{\alpha}+\rho \iint \chi d \xi d \eta \ddot{u}_{s}+\rho \iint \eta \chi d \xi d \eta \ddot{\varphi}_{\xi}-\rho \iint \xi \chi d \xi d \eta \ddot{\varphi}_{\eta}=\left(\iint \chi \sigma_{s} d \xi d \eta\right)^{\prime} \\
& \quad-k_{s} \iint\left[\left(\frac{\partial \chi}{\partial \xi}\right) \eta-\left(\frac{\partial \chi}{\partial \eta}\right) \xi\right] \sigma_{s} d \xi d \eta-\iint\left\{\left[\frac{\partial \chi}{\partial \xi}+k_{\eta} \chi\right] \tau_{s \xi}+\left(\frac{\partial \chi}{\partial \eta}\right) \tau_{s \eta}\right\} d \xi d \eta
\end{aligned}
$$

where two dots over the quantities denote the second partial derivative with respect to time $t$ and superscript prime denotes the first partial derivative with respect to arc length $s . Q_{s}, Q_{\xi}, Q_{\eta}, M_{s}, M_{\xi}$ and $M_{\eta}$ are the components of internal force vector and internal moment vector, respectively. Similarly, $u_{s}(s), u_{\xi}(s), u_{\eta}(s), \varphi_{s}(s), \varphi_{\xi}(s)$ and $\varphi_{\eta}(s)$ are the Frenet components of the displacement and rotational vectors, respectively. Here $\chi(\xi, \eta)$ is the warping function of Saint-Venant's torsion and $\alpha(s)$ is a generalized warping coordinate. $m=\rho A$ is the mass per unite length of the spring, $\rho$ is the mass density of the material, $I_{\xi}$ and $I_{\eta}$ are the second moments of area with respect to $\xi$ and $\eta$ axes, while $I_{P}$ is the polar second moment of area.

For the harmonic motions with circular frequency $\omega$, introducing the following non-dimensional quantities:

$$
\hat{Q}_{i}=\frac{c_{0}^{2} Q_{i}}{E I_{\xi}}, \quad \hat{M}_{i}=\frac{c_{0} M_{i}}{E I_{\xi}}, \quad \hat{u}_{i}=\frac{u_{i}}{c_{0}}, \quad \hat{\varphi}_{i}=\varphi_{i}, \quad \hat{T}=\frac{c_{0}^{2} T}{E \Gamma}, \quad \hat{\alpha}=c_{0} \alpha, \quad(i=s, \xi, \eta)
$$

where $T=\iint \sigma \chi d \xi d \eta$ is the generalized warping moment and the fixed reference value, $R_{0}$, which can be selected arbitrarily, has been used for obtaining the differential equations in non-dimensional form

$$
h_{0}=R_{0} \tan \bar{\alpha}, \quad c_{0}=\sqrt{R_{0}^{2}+h_{0}^{2}}
$$

Expressing the stress components in Eq. (14) and $T=\iint \sigma \chi d \xi d \eta$, and the equivalent constitutive equations of naturally curved and twisted beams [22] in terms of six displacement functions and the generalized warping coordinate, and then combining the results with Eqs (8)-(13) constitutes the non-dimensional free vibration equations for non-cylindrical helical springs including the warping effect

$$
\begin{aligned}
& \frac{d}{d \beta} u_{s}^{0}(\beta)=\frac{R(\beta)}{c(\beta)} u_{\xi}^{0}(\beta)+\frac{c(\beta) I_{\xi}}{c_{0}^{3} A} Q_{s}^{0}(\beta)-\frac{h(\beta) D}{c(\beta) c_{0}^{2} A} \alpha^{0}(\beta) \\
& \frac{d}{d \beta} u_{\xi}^{0}(\beta)=\frac{h(\beta)}{c(\beta)} u_{\eta}^{0}(\beta)-\frac{R(\beta)}{c(\beta)} u_{s}^{0}(\beta)+\frac{c(\beta)}{c_{0}} \varphi_{\eta}^{0}(\beta)+\frac{c(\beta) E I_{\xi}}{c_{0}^{3} G_{\xi} G A} Q_{\xi}^{0}(\beta)
\end{aligned}
$$




$$
\begin{aligned}
\frac{d}{d \beta} u_{\eta}^{0}(\beta) & =-\frac{h(\beta)}{c(\beta)} u_{\xi}^{0}(\beta)-\frac{c(\beta)}{c_{0}} \varphi_{\xi}^{0}(\beta)+\frac{c(\beta) E I_{\xi}}{c_{0}^{3} G_{\eta} G A} Q_{\eta}^{0}(\beta)-\frac{c(\beta) D_{2}}{c_{0}^{2} G_{\eta} A} \alpha^{0}(\beta) \\
\frac{d}{d \beta} \varphi_{s}^{0}(\beta) & =\frac{R(\beta)}{c(\beta)} \varphi_{\xi}^{0}(\beta)+\frac{c(\beta) E I_{\xi}}{c_{0} G I_{P}} M_{s}^{0}(\beta)+\left(\frac{c(\beta) D}{c_{0} I_{P}}+\frac{R(\beta) D_{4}}{c(\beta) c_{0} I_{p}}\right) \alpha^{0}(\beta) \\
\frac{d}{d \beta} \varphi_{\xi}^{0}(\beta) & =\frac{h(\beta)}{c(\beta)} \varphi_{\eta}^{0}(\beta)-\frac{R(\beta)}{c(\beta)} \varphi_{s}^{0}(\beta)+\frac{c(\beta) I_{\xi} \Gamma}{c_{0}\left(I_{\xi} \Gamma-D_{4}^{2}\right)} M_{\xi}^{0}(\beta)-\frac{c(\beta) D_{4} \Gamma}{c_{0}^{2}\left(I_{\xi} \Gamma-D_{4}^{2}\right)} T^{0}(\beta) \\
& +\frac{h(\beta) D_{4} D_{9}}{c(\beta) c_{0}\left(I_{\xi} \Gamma-D_{4}^{2}\right)} \alpha^{0}(\beta)
\end{aligned}
$$

$\frac{d}{d \beta} \varphi_{\eta}^{0}(\beta)=-\frac{h(\beta)}{c(\beta)} \varphi_{\xi}^{0}(\beta)+\frac{c(\beta) I_{\xi}}{c_{0} I_{\eta}} M_{\eta}^{0}(\beta)+\frac{h(\beta) D_{5}}{c(\beta) c_{0} I_{\eta}} \alpha^{0}(\beta)$

$$
\frac{d}{d \beta} \alpha^{0}(\beta)=-\frac{c(\beta) D_{4} I_{\xi}}{\left(I_{\xi} \Gamma-D_{4}^{2}\right)} M_{\xi}^{0}(\beta)+\frac{c(\beta) I_{\xi} \Gamma}{c_{0}\left(I_{\xi} \Gamma-D_{4}^{2}\right)} T^{0}(\beta)+\frac{h(\beta)\left(D_{4} D_{6}-I_{\xi} D_{9}\right)}{c(\beta)\left(I_{\xi} \Gamma-D_{4}^{2}\right)} \alpha^{0}(\beta)
$$

$\frac{d}{d \beta} Q_{s}^{0}(\beta)=\frac{R(\beta)}{c(\beta)} Q_{\xi}^{0}(\beta)-\frac{c(\beta) c_{0}^{3} \rho A \omega^{2}}{E I_{\xi}} u_{s}^{0}(\beta)$

$\frac{d}{d \beta} Q_{\xi}^{0}(\beta)=\frac{h(\beta)}{c(\beta)} Q_{\eta}^{0}(\beta)-\frac{R(\beta)}{c(\beta)} Q_{s}^{0}(\beta)-\frac{c(\beta) c_{0}^{3} \rho A \omega^{2}}{E I_{\xi}} u_{\xi}^{0}(\beta)$

$$
\frac{d}{d \beta} Q_{\eta}^{0}(\beta)=-\frac{h(\beta)}{c(\beta)} Q_{\xi}^{0}(\beta)-\frac{c(\beta) c_{0}^{3} \rho A \omega^{2}}{E I_{\xi}} u_{\eta}^{0}(\beta)
$$

$$
\frac{d}{d \beta} M_{s}^{0}(\beta)=\frac{R(\beta)}{c(\beta)} M_{\xi}^{0}(\beta)-\frac{c(\beta) c_{0} \rho I_{P} \omega^{2}}{E I_{\xi}} \varphi_{s}^{0}(\beta)
$$

$\frac{d}{d \beta} M_{\xi}^{0}(\beta)=\frac{h(\beta)}{c(\beta)} M_{\eta}^{0}(\beta)-\frac{R(\beta)}{c(\beta)} M_{s}^{0}(\beta)+\frac{c(\beta)}{c_{0}} Q_{\eta}^{0}(\beta)-\frac{c(\beta) c_{0} \rho \omega^{2}}{E} \varphi_{\xi}^{0}(\beta)-\frac{c(\beta) \rho D_{4} \omega^{2}}{E I_{\xi}} \alpha^{0}(\beta)$

$\frac{d}{d \beta} M_{\eta}^{0}(\beta)=-\frac{h(\beta)}{c(\beta)} M_{\xi}^{0}(\beta)-\frac{c(\beta)}{c_{0}} Q_{\xi}^{0}(\beta)-\frac{c(\beta) c_{0} \rho I_{\eta} \omega^{2}}{E I_{\xi}} \varphi_{\eta}^{0}(\beta)$

$\frac{d}{d \beta} T^{0}(\beta)=\frac{c(\beta) D_{2} I_{\xi}}{G_{\eta} \Gamma A} Q_{\eta}^{0}(\beta)+\frac{h(\beta) D I_{\xi}}{c(\beta) \Gamma A} Q_{s}^{0}(\beta)-\frac{c_{0} h(\beta) D_{4} D_{9} I_{\xi}}{c(\beta) \Gamma\left(I_{\xi} \Gamma-D_{4}^{2}\right)} M_{\xi}^{0}(\beta)-\frac{c_{0} h(\beta) D_{5} I_{\xi}}{c(\beta) \Gamma I_{\eta}} M_{\eta}^{0}(\beta)$

$-\frac{c_{0} I_{\xi}\left(c^{2}(\beta) D+R(\beta) D_{4}\right)}{c(\beta) \Gamma I_{P}} M_{s}^{0}(\beta)+\frac{h(\beta) D_{9} I_{\xi}}{c(\beta)\left(I_{\xi} \Gamma-D_{4}^{2}\right)} T^{0}(\beta)-\frac{c_{0}}{\Gamma}\left(\frac{h^{2}(\beta) D^{2}}{c(\beta)^{3} A}+\frac{h^{2}(\beta) D_{5}^{2}}{c(\beta)^{3} I_{\eta}}+\right.$ $\frac{h^{2}(\beta) D_{9}\left(I_{\xi} D_{9}-D_{4} D_{6}\right)}{c^{3}(\beta)\left(I_{\xi} \Gamma-D_{4}^{2}\right)}-\frac{h^{2}(\beta) D_{7}}{c^{3}(\beta)}+\frac{R^{2}(\beta) G D_{4}^{2}}{c^{3}(\beta) E I_{P}}-\frac{R^{2}(\beta) G \Gamma}{c(\beta)^{3} E}+\frac{2 R(\beta) G D D_{4}}{c(\beta) E I_{P}}+\frac{c(\beta) G D^{2}}{E I_{P}}$

$$
\left.+\frac{c(\beta) G D_{2}^{2}}{G_{\eta} E A}-\frac{c(\beta) G D_{8}}{E}\right) \alpha^{0}(\beta)-\frac{c(\beta) c_{0} \rho \omega^{2}}{E} \alpha^{0}(\beta)-\frac{c(\beta) c_{0}^{2} \rho D_{4} \omega^{2}}{E \Gamma} \varphi_{\xi}^{0}(\beta)
$$

in which

$$
D=\iint\left[\left(\frac{\partial \varphi}{\partial \xi}\right) \eta-\left(\frac{\partial \varphi}{\partial \eta}\right) \xi\right] d \xi d \eta, D_{2}=\iint \frac{\partial \varphi}{\partial \eta} d \xi d \eta, D_{4}=\iint \eta \varphi d \xi d \eta,
$$




$$
\begin{aligned}
& D_{5}=\iint \xi\left[\left(\frac{\partial \varphi}{\partial \xi}\right) \eta-\left(\frac{\partial \varphi}{\partial \eta}\right) \xi\right] d \xi d \eta, D_{6}=\iint \eta\left[\left(\frac{\partial \varphi}{\partial \xi}\right) \eta-\left(\frac{\partial \varphi}{\partial \eta}\right) \xi\right] d \xi d \eta \\
& D_{7}=\iint\left[\left(\frac{\partial \varphi}{\partial \xi}\right) \eta-\left(\frac{\partial \varphi}{\partial \eta}\right) \xi\right]^{2} d \xi d \eta, D_{8}=\iint\left[\left(\frac{\partial \varphi}{\partial \xi}\right)^{2}+\left(\frac{\partial \varphi}{\partial \eta}\right)^{2}+2 k_{\eta} \varphi\left(\frac{\partial \varphi}{\partial \xi}\right)\right] d \xi d \eta, \\
& D_{9}=\iint \varphi\left[\left(\frac{\partial \varphi}{\partial \xi}\right) \eta-\left(\frac{\partial \varphi}{\partial \eta}\right) \xi\right] d \xi d \eta, \Gamma=\iint \varphi^{2} d \xi d \eta
\end{aligned}
$$

\section{Evaluation of element differential matrix}

Equations (17)-(30) can be written in matrix form as

$$
\frac{d}{d \beta} \mathbf{S}(\beta)=\mathbf{D}(\beta, \omega) \mathbf{S}(\beta)
$$

where $\mathbf{D}(\beta, \omega)$ is the $14 \times 14$ differential matrix and $\mathbf{S}(\beta)$ is the $14 \times 1$ column vector generally known as the state vector. The elements of $\mathbf{S}(\beta)$ are

$$
\mathbf{S}(\beta)=\left\{u_{s}^{0}, u_{\xi}^{0}, u_{\eta}^{0}, \varphi_{s}^{0}, \varphi_{\xi}^{0}, \varphi_{\eta}^{0}, \alpha^{0}, Q_{s}^{0}, Q_{\xi}^{0}, Q_{\eta}^{0}, M_{s}^{0}, M_{\xi}^{0}, M_{\eta}^{0}, T^{0}\right\}^{T}
$$

Now let a non-cylindrical helical spring be divided into $N$ uniform elements, the state vector over the uniform element $i$ between sections: $\beta=\beta_{i}$ and $\beta=\beta_{i+1}$ can be written as

$$
\mathbf{S}_{i+1}(\beta)=e^{\int_{\beta_{i}}^{\beta_{i+1}} \mathbf{D}_{14 \times 14}(\beta, \omega) d \beta} \mathbf{S}_{i}(\beta)=\mathbf{F}_{i}(\omega) \mathbf{S}_{i}(\beta)
$$

For each uniform element, Eq. (31) is showed a strong rigidity, therefore, it is no longer valid evaluating the element transfer matrix with aid of the transfer matrix method [7]. This paper adopts the Scaling and Squaring method and Pad'e approximation [20] to calculate the exponential of this matrix $\mathbf{F}_{i}(\omega)$.

Appling the Scaling and Squaring method to a matrix $\mathbf{A}$, for any positive integer $m$, yields

$$
e^{\mathbf{A}}=\left(e^{\mathbf{A} / m}\right)^{m}
$$

The solution of $e^{\mathbf{A}}$ can be transformed into a problem for evaluating $e^{\mathbf{A} / m}$, and then $e^{\mathbf{A} / m}$ is solved using the Pad'e approximation which, to $e^{\mathbf{B}}$, is defined by

$$
e^{\mathbf{B}}=D_{p q}^{-1}(\mathbf{B}) N_{p q}(\mathbf{B})
$$

where

$$
D_{p q}(\mathbf{B})=\sum_{j=0}^{q} \frac{(p+q-j) ! q !}{(p+q) ! j !(q-j) !}(-\mathbf{B})^{j}
$$

and

$$
N_{p q}(\mathbf{B})=\sum_{j=0}^{p} \frac{(p+q-j) ! p !}{(p+q) ! j !(p-j) !} \mathbf{B}^{j}
$$




\section{Improved Riccati transfer matrix method}

After the element transfer matrix is obtained using the Scaling and Squaring method and Pad'e approximation, the state vector consisting of the 14 elements in Eq. (31) can be decomposed into two state vectors and each of them has 7 elements $[18,19]$, that is,

$$
\mathbf{S}_{14 \times 1}(\beta)=\left\{u_{s}^{0}, u_{\xi}^{0}, u_{\eta}^{0}, \phi_{s}^{0}, \phi_{\xi}^{0}, \phi_{\eta}^{0}, \alpha^{0}, Q_{s}^{0}, Q_{\xi}^{0}, Q_{\eta}^{0}, M_{s}^{0}, M_{\xi}^{0}, M_{\eta}^{0}, T^{0}\right\}^{T}=\left\{\mathbf{f}_{7 \times 1}, \mathbf{e}_{7 \times 1}\right\}
$$

where the vectors $\mathbf{f}_{7 \times 1}$ and $\mathbf{e}_{7 \times 1}$ must satisfy the boundary conditions at the initial section $(\beta=0)$ of the spring. It follows that Eq. (38) becomes for the spring with clamped-clamped ends

$$
\mathbf{f}_{7 \times 1}=\mathbf{0}, \quad \mathbf{e}_{7 \times 1} \neq \mathbf{0}
$$

and the element transfer relationship can be rewritten as

$$
\left\{\begin{array}{l}
\mathbf{f} \\
\mathbf{e}
\end{array}\right\}_{i+1}=\left[\begin{array}{l}
\mathbf{F}_{11}(\omega) \mathbf{F}_{12}(\omega) \\
\mathbf{F}_{21}(\omega) \mathbf{F}_{22}(\omega)
\end{array}\right]_{i}\left\{\begin{array}{l}
\mathbf{f} \\
\mathbf{e}
\end{array}\right\}_{i}
$$

Expanding the above equation gives

$$
\begin{gathered}
\mathbf{f}_{i+1}=\mathbf{F}_{11}(\omega)_{i} \mathbf{f}_{i}+\mathbf{F}_{12}(\omega)_{i} \mathbf{e}_{i} \\
\mathbf{e}_{i+1}=\mathbf{F}_{21}(\omega)_{i} \mathbf{f}_{i}+\mathbf{F}_{22}(\omega)_{i} \mathbf{e}_{i}
\end{gathered}
$$

Introducing the Riccati transform, $\mathbf{f}_{i}=\mathbf{S}_{i} \mathbf{e}_{i}$, and substituting it into Eqs (41) and (42) yields

$$
\begin{aligned}
& \mathbf{f}_{i+1}=\left[\mathbf{F}_{11}(\omega) \mathbf{S}+\mathbf{F}_{12}(\omega)\right]_{i}\left[\mathbf{F}_{21}(\omega) \mathbf{S}+\mathbf{F}_{22}(\omega)\right]_{i}^{-1} \mathbf{e}_{i+1} \\
& \mathbf{e}_{i}=\left[\mathbf{F}_{21}(\omega) \mathbf{S}+\mathbf{F}_{22}(\omega)\right]_{i}^{-1} \mathbf{e}_{i+1}
\end{aligned}
$$

Referring to Eqs (43) and (44), the following Riccati transform recursive relationship can be obtained

$$
\mathbf{S}_{i+1}=\left[\mathbf{F}_{11}(\omega) \mathbf{S}+\mathbf{F}_{12}(\omega)\right]_{i}\left[\mathbf{F}_{21}(\omega) \mathbf{S}+\mathbf{F}_{22}(\omega)\right]_{i}^{-1}
$$

Obviously, we find $\mathbf{S}_{1}=\mathbf{0}$ from the boundary conditions at the initial section of the spring. Thus, for the Nth uniform element, we may write

$$
\mathbf{f}_{N+1}=\mathbf{S}_{N+1} \mathbf{e}_{N+1}
$$

which leads directly to different frequency equations from the boundary conditions at the end $\operatorname{section}(\beta=2 n \pi)$ for the different types of springs. For example, when the springs have the same boundary conditions at both ends, the frequency equation is

$$
\left|\mathbf{S}_{N+1}\right| \prod_{i=1}^{N} \operatorname{sign}\left|\mathbf{F}_{21}(\omega) \mathbf{S}+\mathbf{F}_{22}(\omega)\right|_{i}=\mathbf{0}
$$

From which the natural frequencies and vibrating mode shapes for non-cylindrical helical springs with clampedclamped ends can be determined.

\section{Results and discussion}

In order to demonstrate the effectiveness of the method presented in this paper, three numerical examples are given and the results are discussed. Some material and geometrical properties are given as $E=206 \mathrm{GPa}, \mu=0.3, \rho=$ $7850 \mathrm{~kg} / \mathrm{m}^{3}$, the helix pitch angle $\bar{\alpha}$, the number of active turns $n$, maximum cylinder radius $R_{1}$ and the minimum cylinder radius $R_{2}$ (for conical and barrel-types helical springs), maximum cylinder radius $R_{2}$ and the minimum cylinder radius $R_{1}$ (for hyperboloidal-type helical spring). 
Table 1

First five natural frequencies of conical type spring with the elliptic cross-section

\begin{tabular}{crcccc}
\hline Mode no. & FEM & \multicolumn{4}{c}{ Present study(PS) } \\
\cline { 3 - 6 } & & Warping ignored & Errors (\%) & Warping included & Errors (\%) \\
\hline 1 & 53.16 & 76.58 & 44.06 & 53.43 & 0.51 \\
2 & 73.68 & 83.16 & 12.87 & 74.77 & 1.48 \\
3 & 74.16 & 84.82 & 14.37 & 75.17 & 1.35 \\
4 & 101.88 & 111.72 & 9.66 & 102.48 & 0.59 \\
5 & 106.98 & 143.30 & 33.95 & 111.39 & 4.12 \\
\hline
\end{tabular}

Table 2

First five natural frequencies of hyperboloidal type spring with the elliptic cross-section $\left(R_{1}=15 \mathrm{~mm}, R_{2}=25 \mathrm{~mm}\right)$

\begin{tabular}{cccccc}
\hline Mode no. & FEM & \multicolumn{4}{c}{ Present study(PS) } \\
\cline { 3 - 5 } & & Warping ignored & Errors (\%) & Warping included & Errors (\%) \\
\hline 1 & 55.73 & 79.83 & 43.24 & 55.94 & 0.38 \\
2 & 85.90 & 96.93 & 12.84 & 87.03 & 1.32 \\
3 & 86.51 & 97.98 & 13.26 & 87.61 & 1.27 \\
4 & 123.70 & 149.16 & 20.58 & 124.33 & 0.51 \\
5 & 142.42 & 169.07 & 18.71 & 148.23 & 4.08 \\
\hline
\end{tabular}

Table 3

First five natural frequencies of barrel type spring with the elliptic cross-section

\begin{tabular}{cccccc}
\hline Mode no. & FEM & \multicolumn{4}{c}{ Present study(PS) } \\
\cline { 3 - 6 } & & Warping ignored & Errors (\%) & Warping included & Errors (\%) \\
\hline 1 & 48.05 & 68.67 & 42.91 & 47.78 & 0.62 \\
2 & 64.00 & 72.06 & 12.59 & 64.36 & 0.56 \\
3 & 64.14 & 72.44 & 12.94 & 64.53 & 0.61 \\
4 & 85.78 & 89.40 & 4.22 & 88.39 & 3.04 \\
5 & 88.86 & 124.83 & 40.48 & 89.23 & 0.42 \\
\hline
\end{tabular}

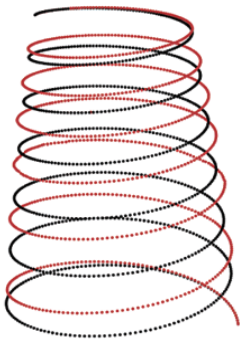

(a)

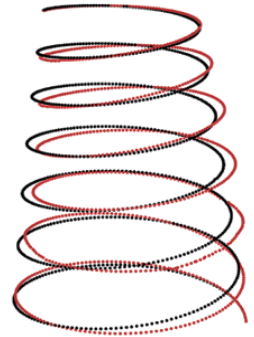

(b)

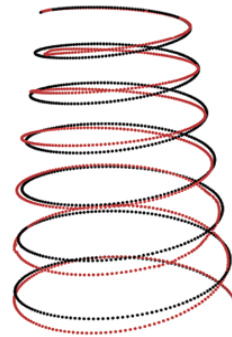

(c)

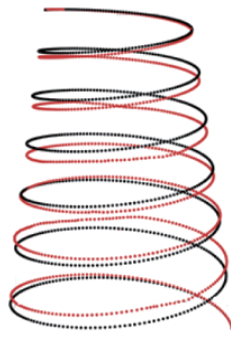

(d)

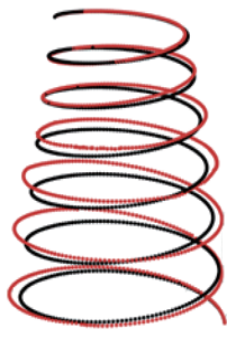

(e)

Fig. 3. First five mode shapes of the free vibrating conical type spring with the elliptic cross-section: (a) First mode (b) Second mode (c) Third mode (d) Fourth mode (e) Fifth mode.

\subsection{Example 1}

As an example, the three types of springs with elliptic cross-section and clamped-clamped ends, and with geometry properties: $R_{1}=25 \mathrm{~mm}, R_{2}=15 \mathrm{~mm}, n=6.5, \bar{\alpha}=4.8^{\circ}$, the semi-axe $a=1.5 \mathrm{~mm}$ in the $\xi$-direction and semi-axe $b=0.6 \mathrm{~mm}$ in the $\eta$-direction, shape factors $G_{\xi}=0.91$ and $G_{\eta}=0.81$, are considered first. A general-purpose computer program is coded in MATHEMATICA to perform the free vibration analysis of the springs. The warping function of the cross-section is [23]

$$
\chi(\xi, \eta)=-\frac{a^{2}-b^{2}}{a^{2}+b^{2}} \xi \eta
$$

The first five natural frequencies $f=\omega / 2 \pi$ measured in $\mathrm{Hz}$ for three types of springs calculated by using the program coded in this study, with and without an allowance for warping, are given in Tables 1-3, together with those 


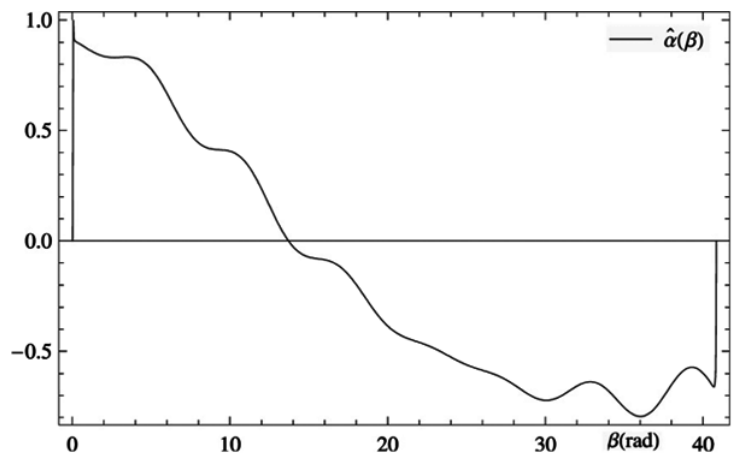

(a)

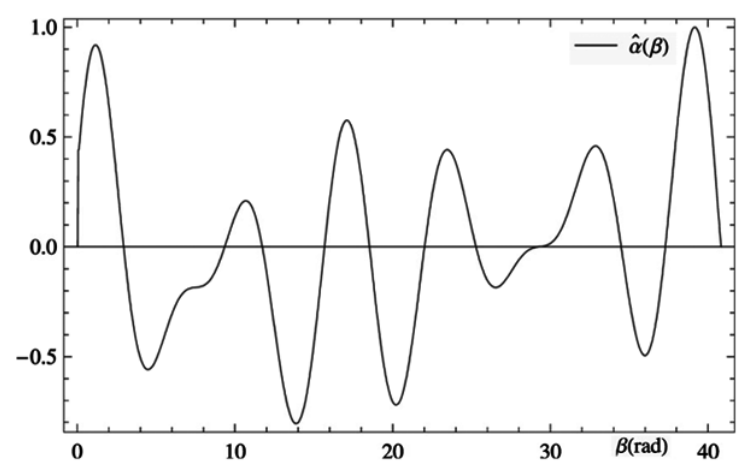

(c)

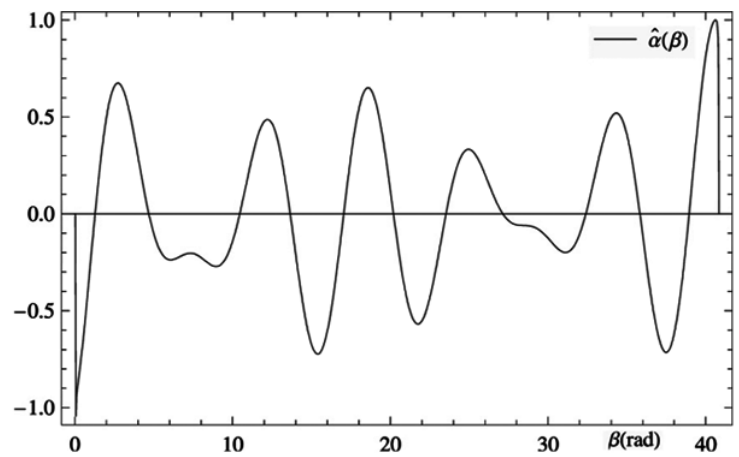

(b)

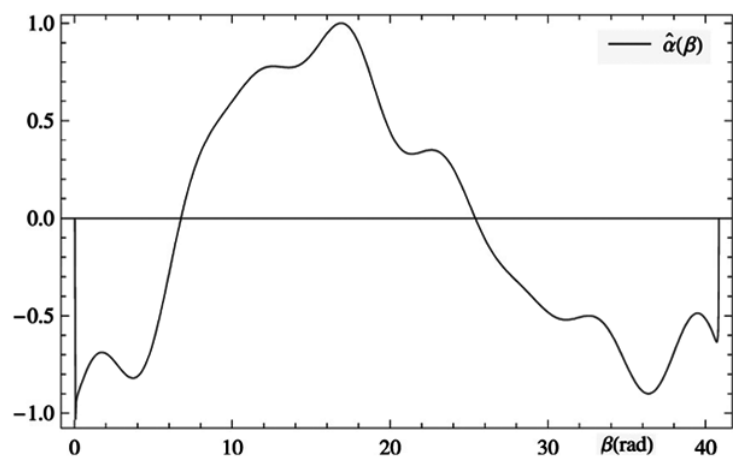

(d)

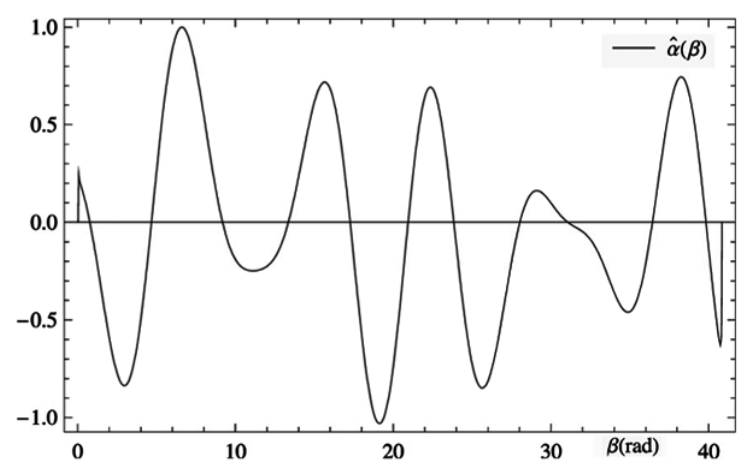

(e)

Fig. 4. Normal mode shapes of the generalized warping coordinate: (a) First mode (53.43 Hz) (b) Second mode (74.77 Hz) (c) Third mode $(75.17 \mathrm{~Hz})(\mathrm{d})$ Fourth mode $(102.48 \mathrm{~Hz})(\mathrm{e})$ Fifth mode $(111.39 \mathrm{~Hz})$.

obtained from ANSYS. To analyze these springs using the finite element method (FEM), we partition them into 6000 three-dimensional solid elements (Solid45), and the total number of nodal points is 10217 . The first five mode shapes of the conical spring are shown in Fig. 3. Modes 2 and 3 are predominantly transverse vibrational motion and modes 1 and 4 are predominantly axial vibrational motion. The remaining mode is predominantly torsional vibrational motion in character. The first five normal mode shapes showing the generalized warping coordinate are presented in the local coordinate system, as shown in Fig. 4. It is interesting to see from the plots that the normal mode shapes of the conical springs, different from those of hyperboloidal, barrel and cylindrical [11] helical springs, are neither symmetric nor antisymmetric about the midpoint under given boundary conditions.

These results of Tables 1-3 demonstrate that the effect of warping deformation on the natural frequencies of non-cylindrical helical springs with the elliptic cross-section is similar to that encountered in cylindrical helical 
Table 4

First five natural frequencies of conical type spring with four cross-sectional shapes $\left(R_{1}=25 \mathrm{~mm}, R_{2}=15 \mathrm{~mm}\right)$

\begin{tabular}{|c|c|c|c|c|c|c|}
\hline \multicolumn{2}{|c|}{ Mode no. } & 1 & 2 & 3 & 4 & 5 \\
\hline Square & FEM & 70.22 & 75.66 & 86.63 & 88.78 & 133.99 \\
\hline $\begin{array}{l}2 a=2 b= \\
1.773 \mathrm{~mm}\end{array}$ & PS & 64.67 & 75.38 & 84.63 & 86.51 & 124.42 \\
\hline $\begin{array}{l}\text { Circle } \\
r=1 \mathrm{~mm}\end{array}$ & Literature [4] & 69.21 & 74.44 & 85.24 & 87.43 & 131.98 \\
\hline Rectangle & FEM & 64.13 & 86.60 & 88.09 & 100.08 & 123.25 \\
\hline $\begin{array}{l}2 a=2.288 \mathrm{~mm} \\
2 b=1.373 \mathrm{~m} \mathrm{~m}\end{array}$ & PS & 60.32 & 85.55 & 86.04 & 100.13 & 117.61 \\
\hline Ellipse & FEM & 63.21 & 83.35 & 85.12 & 94.70 & 121.11 \\
\hline $\begin{array}{l}2 a=2.577 \mathrm{~mm} \\
2 b=1.552 \mathrm{~mm}\end{array}$ & PS & 64.33 & 85.62 & 86.94 & 98.11 & 123.23 \\
\hline $\begin{array}{l}\text { Equilateral } \\
\text { triangle }\end{array}$ & FEM & 61.74 & 80.93 & 87.55 & 90.37 & 119.20 \\
\hline$b=0.777 \mathrm{~mm}$ & PS & 58.97 & 80.02 & 85.90 & 88.76 & 113.04 \\
\hline Rectangle & FEM & 58.68 & 64.23 & 73.48 & 75.73 & 113.37 \\
\hline $\begin{array}{l}2 a=1.373 \mathrm{~mm} \\
2 b=2.288 \mathrm{~mm}\end{array}$ & PS & 58.38 & 61.13 & 72.81 & 74.90 & 112.97 \\
\hline Ellipse & FEM & 56.71 & 63.29 & 71.18 & 73.35 & 109.59 \\
\hline $\begin{array}{l}2 a=1.552 \mathrm{~mm} \\
2 b=2.577 \mathrm{~mm}\end{array}$ & PS & 57.59 & 64.40 & 72.51 & 74.46 & 111.13 \\
\hline
\end{tabular}

springs with the same cross-sectional shape [11]. However, the effect is much more pronounced in the case of non-cylindrical helical springs than that of cylindrical helical springs. The necessity to account for the warping effect when the natural frequencies in the springs with noncircular cross-sections are required is quite clear. It is clearly evident that the results of the present model demonstrate a good agreement with those of ANSYS for the case when the warping effect is included. It may be noted from the tables that for the three types of springs with the elliptical cross-section, the frequencies associated with both the second and third modes become close to each other, which is different from the barrel type of spring with the circle cross-section and the same boundary condition, whose third and forth frequencies are very close in value [3,7,24]. Furthermore, from the tables, as seen, when the number of modes of the springs increases the difference among the frequencies corresponding to these modes also increases.

Tables 1-3 also show that when the material properties, the radii of the maximum cylinder and the minimum cylinder, the helix pitch angle, the number of active turns and the cross-section area are the same, the hyperboloidal spring has the largest rigidity compared to other types of springs and the conical spring follows the hyperboloidal type with respect to rigidity. Therefore, the hyperboloidal spring gives the highest frequencies and the barrel spring gives the lowest ones. While a barrel-type spring has very close natural frequencies compared to other types.

\subsection{Example 2}

In order to compare with the results given from ANSYS and the scientific and technical literature [7,24], the first five natural frequencies, for the three types of springs with four cross- sectional shapes and clamped-clamped ends, are computed when the spring's cross-sectional areas keep fixed.

In this example, both $\bar{\alpha}=4.8^{\circ}$ and $n=6.5$ are the same for all the springs and modulus of elasticity is $E=$ $210 \mathrm{GPa}$. Assuming that the radius of the circular cross-section is denoted by $r$; the semi-axes both in the $\xi-$ and $\eta$ - directions of the elliptic cross-section are respectively denoted by $a$ and $b$; the width and the height both in the $\xi$ - and $\eta$-directions of the rectangular cross-section are respectively denoted by $2 a$ and $2 b$, and its shape factor is $G_{\xi}=G_{\eta}=0.842$. The warping function is [23]

$$
\varphi(\xi, \eta)=\xi \eta-\frac{32 a^{2}}{\pi^{3}} \sum_{n=0}^{\infty} \frac{(-1)^{n} \sinh \frac{(2 n+1) \pi \eta}{2 a}}{(2 n+1)^{3} \cosh \frac{(2 n+1) \pi b}{2 a}} \sin \frac{(2 n+1) \pi \xi}{2 a}
$$

and assuming that the altitude in the $\xi$-direction of the uniform equilateral triangle cross- section is denoted by $3 b$, and the shape factor is $G_{\xi}=G_{\eta}=0.757$. The warping function is [23] 
Table 5

First five natural frequencies of hyperboloidal type spring with four cross-sectional shapes $\left(R_{1}=15 \mathrm{~mm}, R_{2}=25 \mathrm{~mm}\right)$

\begin{tabular}{lcccccc}
\hline \multicolumn{1}{c}{ Mode no. } & & 1 & 2 & 3 & 4 & 5 \\
\hline Square & FEM & 73.23 & 90.02 & 96.44 & 110.95 & 152.50 \\
$2 a=2 b=1.773 \mathrm{~mm}$ & PS & 67.81 & 89.17 & 94.68 & 109.83 & 146.15 \\
Circle & Literature [15] & 71.68 & 88.70 & 94.41 & 109.54 & 150.51 \\
$r=1 \mathrm{~mm}$ & & & & & & \\
Rectangle & FEM & 67.11 & 98.82 & 100.92 & 134.39 & 148.12 \\
$2 a=2.288 \mathrm{~mm}$ & PS & 63.80 & 97.75 & 98.94 & 134.42 & 11.49 \\
$2 b=1.373 \mathrm{~mm}$ & & & & & & \\
Ellipse & FEM & 66.17 & 95.09 & 97.30 & 127.11 & 145.31 \\
$2 a=2.577 \mathrm{~mm}$ & PS & 67.31 & 97.62 & 99.44 & 131.68 & 147.56 \\
$2 b=1.552 \mathrm{~mm}$ & & & & & & \\
Equilateral triangle & FEM & 64.86 & 94.37 & 98.70 & 117.64 & 142.88 \\
$b=0.777 \mathrm{~mm}$ & PS & 61.68 & 92.90 & 96.44 & 115.82 & 136.75 \\
Rectangle & FEM & 67.10 & 72.41 & 80.86 & 90.44 & 125.86 \\
$2 a=1.373 \mathrm{~mm}$ & PS & 63.77 & 72.79 & 80.91 & 89.80 & 125.12 \\
$2 b=2.288 \mathrm{~mm}$ & & & & & & \\
Ellipse & FEM & 66.13 & 69.95 & 78.18 & 87.42 & 121.69 \\
$2 a=1.552 \mathrm{~mm}$ & PS & 67.35 & 71.02 & 79.38 & 86.66 & 123.73 \\
$2 b=2.577 \mathrm{~mm}$ & & & & & & \\
\hline
\end{tabular}

Table 6

First five natural frequencies of barrel type spring with four cross-sectional shapes $\left(R_{1}=\right.$ $\left.25 \mathrm{~mm}, R_{2}=15 \mathrm{~mm}\right)$

\begin{tabular}{lcccccc}
\hline \multicolumn{1}{c}{ Mode no. } & & 1 & 2 & 3 & 4 & 5 \\
\hline Square & FEM & 61.45 & 63.76 & 77.26 & 77.39 & 117.65 \\
$2 a=2 b=1.773 \mathrm{~mm}$ & PS & 57.68 & 61.49 & 74.87 & 75.10 & 107.74 \\
Circle & Literature [4] & 59.63 & 61.78 & 75.08 & 75.09 & 114.01 \\
$r=1 \mathrm{~mm}$ & & & & & & \\
Rectangle & FEM & 57.93 & 76.30 & 76.79 & 80.43 & 107.46 \\
$2 a=2.288 \mathrm{~mm}$ & PS & 54.41 & 73.83 & 74.14 & 79.88 & 101.72 \\
$2 b=1.373 \mathrm{~mm}$ & & & & & & \\
Ellipse & FEM & 54.76 & 66.35 & 70.42 & 70.70 & 101.62 \\
$2 a=2.577 \mathrm{~mm}$ & PS & 57.61 & 74.93 & 75.15 & 78.02 & 106.12 \\
$2 b=1.552 \mathrm{~mm}$ & & & & & & \\
Equilateral triangle & FEM & 55.71 & 67.26 & 77.60 & 77.93 & 104.04 \\
$b=0.777 \mathrm{~mm}$ & PS & 52.34 & 66.01 & 74.89 & 77.22 & 98.77 \\
Rectangle & FEM & 47.90 & 58.16 & 67.05 & 67.23 & 97.43 \\
$2 a=1.373 \mathrm{~mm}$ & PS & 47.48 & 54.89 & 65.92 & 66.13 & 96.61 \\
$2 b=2.288 \mathrm{~mm}$ & & & & & & \\
Ellipse & FEM & 46.32 & 54.97 & 63.55 & 63.70 & 94.16 \\
$2 a=1.552 \mathrm{~mm}$ & PS & 46.52 & 57.31 & 64.86 & 66.25 & 94.47 \\
$2 b=2.577 \mathrm{~mm}$ & & & & & & \\
\hline
\end{tabular}

$$
\varphi(\xi, \eta)=\frac{\eta^{3}-3 \xi^{2} \eta}{6 b}
$$

From Tables 4-6, as expected, for the three types of springs with different shapes of cross- section (square, circle, ellipse and rectangular), the natural frequencies depend on the stiffness of the system, the shapes of wire cross-section and the different arrangements of cross-section when the material properties, the radii of the maximum cylinder and the minimum cylinder, the helix pitch angle, the number of active turns and the cross-sectional areas of wire are the same.

\subsection{Example 3}

After verifying the soundness of the frequencies obtained for the above-mentioned springs, effects of vibrational parameters which are chosen as the aspect ratio $a / b$ ranging from 0.4 to 2.5 (here $a$ may be greater than, less than, or equal to, $b$ ), the helix pitch angle $\bar{\alpha}$ from $4^{\circ}$ to $10^{\circ}$, the number of active turns $n$ from 4 to 10 and the ratio of radii 
Table 7

The effect of the ratio $\left(R_{2} / R_{1}\right)$ on frequencies $\left(R_{1}=25 \mathrm{~mm}, \bar{\alpha}=4.8^{\circ}\right.$, $n=6.5,2 a=2.4 \mathrm{~mm}, 2 b=3 \mathrm{~mm})$

\begin{tabular}{|c|c|c|c|c|c|c|}
\hline \multicolumn{2}{|c|}{ Mode no. } & 1 & 2 & 3 & 4 & 5 \\
\hline$R_{1}=25 \mathrm{~mm}$ & FEM & 64.09 & 67.43 & 80.29 & 80.80 & 126.22 \\
\hline$R_{2}=20 \mathrm{~mm}$ & PS & 64.77 & 68.56 & 81.55 & 81.97 & 127.73 \\
\hline$R_{1}=25 \mathrm{~mm}$ & FEM & 70.61 & 79.82 & 93.97 & 94.15 & 143.13 \\
\hline$R_{2}=15 \mathrm{~mm}$ & PS & 71.22 & 80.72 & 95.18 & 95.21 & 144.41 \\
\hline$R_{1}=25 \mathrm{~mm}$ & FEM & 77.45 & 92.86 & 109.10 & 109.21 & 159.09 \\
\hline$R_{2}=10 \mathrm{~mm}$ & PS & 78.08 & 93.69 & 110.33 & 110.39 & 160.17 \\
\hline$R_{1}=25 \mathrm{~mm}$ & FEM & 85.02 & 105.46 & 125.51 & 125.60 & 174.81 \\
\hline$R_{2}=5 \mathrm{~mm}$ & PS & 85.56 & 106.46 & 126.63 & 126.68 & 175.70 \\
\hline
\end{tabular}

Table 8

The effect of helix angle $(\bar{\alpha})$ on frequencies $\left(R_{1}=25 \mathrm{~mm}, R_{2}=\right.$ $15 \mathrm{~mm}, n=6.5,2 a=2.4 \mathrm{~mm}, 2 b=3 \mathrm{~mm}$ )

\begin{tabular}{ccccrrc}
\hline \multicolumn{2}{c}{ Mode no. } & 1 & \multicolumn{1}{c}{2} & \multicolumn{1}{c}{3} & \multicolumn{1}{c}{4} & \multicolumn{1}{c}{5} \\
\hline $\bar{\alpha}=4^{\circ}$ & FEM & 70.85 & 79.70 & 99.36 & 99.48 & 142.78 \\
& PS & 71.31 & 80.78 & 100.27 & 100.39 & 143.83 \\
$\bar{\alpha}=4.8^{\circ}$ & FEM & 70.61 & 79.82 & 93.97 & 94.15 & 143.13 \\
& PS & 71.22 & 80.72 & 95.18 & 95.21 & 144.41 \\
$\bar{\alpha}=6^{\circ}$ & FEM & 70.49 & 79.50 & 86.44 & 86.62 & 142.95 \\
& PS & 70.04 & 80.57 & 87.66 & 87.72 & 144.14 \\
$\bar{\alpha}=8^{\circ}$ & FEM & 69.86 & 74.89 & 75.23 & 79.20 & 141.74 \\
& PS & 70.51 & 76.12 & 76.33 & 80.35 & 143.15 \\
$\bar{\alpha}=10^{\circ}$ & FEM & 64.86 & 65.17 & 69.96 & 78.77 & 138.62 \\
& PS & 65.98 & 66.32 & 70.69 & 79.89 & 140.50 \\
\hline
\end{tabular}

Table 9

The effect of number of active turns $(n)$ on frequencies $\left(R_{1}=25 \mathrm{~mm}, R_{2}=\right.$ $\left.15 \mathrm{~mm}, \bar{\alpha}=4.8^{\circ}, 2 a=2.4 \mathrm{~mm}, 2 b=3 \mathrm{~mm}\right)$

\begin{tabular}{ccrrrrr}
\hline \multicolumn{2}{c}{ Mode no. } & 1 & 2 & 3 & \multicolumn{1}{c}{4} & 5 \\
\hline$n=4$ & FEM & 113.33 & 126.66 & 165.14 & 167.58 & 204.76 \\
& PS & 113.75 & 127.78 & 165.73 & 168.78 & 205.95 \\
$n=6$ & FEM & 76.46 & 85.84 & 103.85 & 104.47 & 154.40 \\
& PS & 77.11 & 87.02 & 104.97 & 105.75 & 155.66 \\
$n=6.5$ & FEM & 70.61 & 79.82 & 93.97 & 94.15 & 143.13 \\
& PS & 71.22 & 80.72 & 95.18 & 95.21 & 144.41 \\
$n=8$ & FEM & 57.60 & 64.65 & 71.10 & 71.47 & 118.52 \\
& PS & 58.16 & 65.62 & 72.07 & 72.49 & 119.67 \\
$n=10$ & FEM & 46.21 & 50.86 & 51.54 & 52.81 & 95.87 \\
& PS & 46.64 & 51.66 & 52.31 & 53.61 & 96.79 \\
\hline
\end{tabular}

of the minimum cylinder to the maximum cylinder $R_{2} / R_{1}$ ranging from 0.2 to 0.8 on the natural frequencies of the barrel spring with the elliptic cross-section and clamped-clamped ends are studied. The same problem has also been done using ANSYS with 6000 three-dimensional solid elements. It can be seen from Tables 7 to 9 that as $R_{2} / R_{1}$, $\bar{\alpha}$ and $n$ increase, the spring length increases and the stiffness of the system decreases, which in turn reduces the frequencies.

The variation of the natural frequencies with respect to the aspect ratio $(a / b)$, is given in Table 10 . From the table, as expected, the natural frequencies increase with increase of the cross-sectional areas of the spring. When the spring's cross-sectional areas keep fixed, the different arrangements of the cross-section will have noticeable effect on the natural frequencies.

\section{Conclusions}

Improved Riccati transfer matrix method for free vibration of non-cylindrical helical springs including warping is presented. To the knowledge of the author, this is the first free vibration analysis of the springs that take into 
Table 10

The effect of the aspect ratio $(a / b)$ on frequencies $\left(R_{1}=25 \mathrm{~mm}, R_{2}=\right.$ $15 \mathrm{~mm}, \bar{\alpha}=4.8^{\circ}, n=6.5$ )

\begin{tabular}{|c|c|c|c|c|c|c|}
\hline \multicolumn{2}{|c|}{ Mode no. } & 1 & 2 & 3 & 4 & 5 \\
\hline $2 a=3 \mathrm{~mm}$ & FEM & 48.05 & 64.00 & 64.14 & 85.78 & 88.86 \\
\hline $2 b=1.2 \mathrm{~mm}$ & PS & 47.78 & 64.36 & 64.53 & 88.39 & 89.23 \\
\hline $2 a=3 \mathrm{~mm}$ & FEM & 65.74 & 84.49 & 85.45 & 87.70 & 121.85 \\
\hline $2 b=1.8 \mathrm{~mm}$ & PS & 66.21 & 85.98 & 86.74 & 89.34 & 122.74 \\
\hline $2 a=3 \mathrm{~mm}$ & FEM & 79.23 & 87.63 & 100.16 & 100.35 & 148.02 \\
\hline $2 b=2.4 \mathrm{~mm}$ & PS & 80.32 & 89.17 & 101.77 & 101.83 & 149.35 \\
\hline $2 a=2.4 \mathrm{~mm}$ & FEM & 70.61 & 79.82 & 93.97 & 94.15 & 143.13 \\
\hline $2 b=3 \mathrm{~mm}$ & PS & 71.22 & 80.72 & 95.18 & 95.21 & 144.41 \\
\hline $2 a=1.8 \mathrm{~mm}$, & FEM & 53.22 & 65.94 & 74.72 & 74.96 & 108.28 \\
\hline $2 b=3 \mathrm{~mm}$ & PS & 53.47 & 66.53 & 75.40 & 75.42 & 108.75 \\
\hline $2 a=1.2 \mathrm{~mm}$ & FEM & 35.77 & 48.23 & 52.35 & 52.51 & 72.82 \\
\hline $2 b=3 \mathrm{~mm}$ & PS & 35.66 & 48.07 & 52.36 & 52.37 & 72.59 \\
\hline
\end{tabular}

consideration the warping effect upon the natural frequencies. The method is free from ill-conditioning system of equations usually associated with complex (numerical) matrix manipulation. The numerical results obtained from the proposed method illustrate quite well that the warping effect can have strong influences on the natural frequencies of such springs. Therefore, it is absolutely necessary to include the warping effect when the natural frequencies of the springs are computed. In future work the current method can be developed further to include the free vibration analysis of symmetric laminated non-cylindrical helical springs made of orthotropic materials for the case when the warping effect is included.

\section{Acknowledgments}

The work was supported by the National Natural Science Foundation of P. R. China (Grant no. 10572105) and Shanghai Leading Academic Discipline Project, Project no. B302.

\section{References}

[1] I. Epsien, The motion of a conical coil spring, Journal of Applied Physics 18 (1947), 368-374.

[2] K. Nagaya, S. Takeda and Y. Nakata, Free vibration of coil spring of arbitrary shape, International Journal for Numerical Metnods in Engineering 23 (1986), 1081-1099.

[3] V. Yildirim, Free vibration analysis of non-cylindrical coil springs by combined used of the transfer matrix and the complementary functions method, Communications in Numerical Methods in Engineering 13 (1997), 487-494.

[4] V. Yildirim, E. Sancaktar and E. Kiral, Effect of the material types on the fundamental frequencies of uniaxial composite conical springs, American Society of Mechanical Engineers, Design Engineering Division (Publication) DE 105 (1999), 37-50.

[5] V. Yildirim, E. Sancaktar and E. Kiral, Fundamental frequencies of uniaxial composite barrel and hyperboloidal springs, American Society of Mechanical Engineers, Design Engineering Division (Publication) DE 105 (1999), 25-35.

[6] V. Yildirim, A parametric study on the natural frequencies of unidirectional composite conical springs, Communications in Numerical Methods in Engineering 20 (2004), 207-227.

[7] V. Yildirim and N. Ince, Natural frequencies of helical springs of arbitrary shape, Journal of Sound and Vibration 204 (1997), $311-329$.

[8] M.H. Wu and W.Y. Hsu, Modelling the static and dynamic behavior of a conical spring by considering the coil close and damping effects, Journal of Sound and Vibration 214 (1998), 17-28.

[9] W. Busool and M. Eisenberger, Free vibration of helicoidal beams of arbitrary shape and variable cross section, ASME Journal of Vibration and Acoustics 124 (2002), 397-409.

[10] F.F. Calım, Dynamic analysis of composite coil springs of arbitrary shape, Composites Part B: Engineering 40 (2009), $741-757$.

[11] A.M. Yu and Y. Hao, Free vibration analysis of cylindrical helical springs with noncircular cross-sections, Journal of Sound and Vibration 330 (2011), 2628-2639.

[12] B. He, X.T. Rui and Y.Q. Lu, Riccati discrete time transfer matrix method for multibody system dynamics, Binggong Xuebao/Acta Armamentarii 27 (2006), 622-625. (in Chinese).

[13] N.G. Stephen, On the Riccati transfer matrix method for repetitive structures, Mechanics Research Communications 37 (2010), $663-665$.

[14] A.M. Ellakany, Calculation of higher natural frequencies of simply supported elastic composite beams using Riccati matrix method, Meccanica 43 (2008), 523-532.

[15] B. He, X.T. Rui and G.P. Wang, Riccati discrete time transfer matrix method for elastic beam undergoing large overall motion, Multibody System Dynamics 18 (2007), 579-598. 
[16] S. Chai, X.Y. Gang and Q.W. Qu, A whole transfer matrix method for the eigensolutions of multi-rotor systems, Proceedings of the ASME Power Conference PART A (2005), 457-463.

[17] Z.P. Gu and S.Q. Chen, CSDT-Riccati transfer matrix integration method for transient analysis of nonlinear rotor-bearing systems, Hangkong Dongli Xuebao/Journal of Aerospace Power 6 (1991), 41-45, (in Chinese).

[18] B.G. Liu, Perturbation RICCATI Transfer Matrix Method for One Dimensional Structure with Parameter Uncertainties and Its Applications, Ph. D. Dissertation, Chongqing University, 2002, (in Chinese).

[19] Z. Wang, The singularity and its eliminating methods of Riccati transfer matrix method, Journal of Vibration and Shock 2 (1987), 74-78, (in Chinese).

[20] C. Moler and C. Van Loan, Nineteen Dubious Ways to Compute the Exponential of a Matrix, Twenty-Five Years Later, Society for Industrial and Applied Mathematics (SIAM) 45 (2003), 3-49.

[21] I.S. Sokolnikoff and R.M. Redheffer, Mathematics of Physics and Modern Engineering, McGraw-Hill: Tokyo, 1958.

[22] A.M. Yu and C.J. Yang, Generalized variational principle of dynamic analysis on naturally curved and twisted box beams for anisotropic materials, Meccanica 43 (2008), 611-622.

[23] C. Wei-Zang, H.-S. Lin, H.-C. Hu and K.-Y. Ye, Torsion Theory of Elastic Cylinder, Science Press, Shanghai, 1956, (in Chinese).

[24] V. Yildirim, A parametric study on the free vibration of non-cylindrical helical springs, ASME Journal of Applied Mechanics 65 (1998), 157-163. 

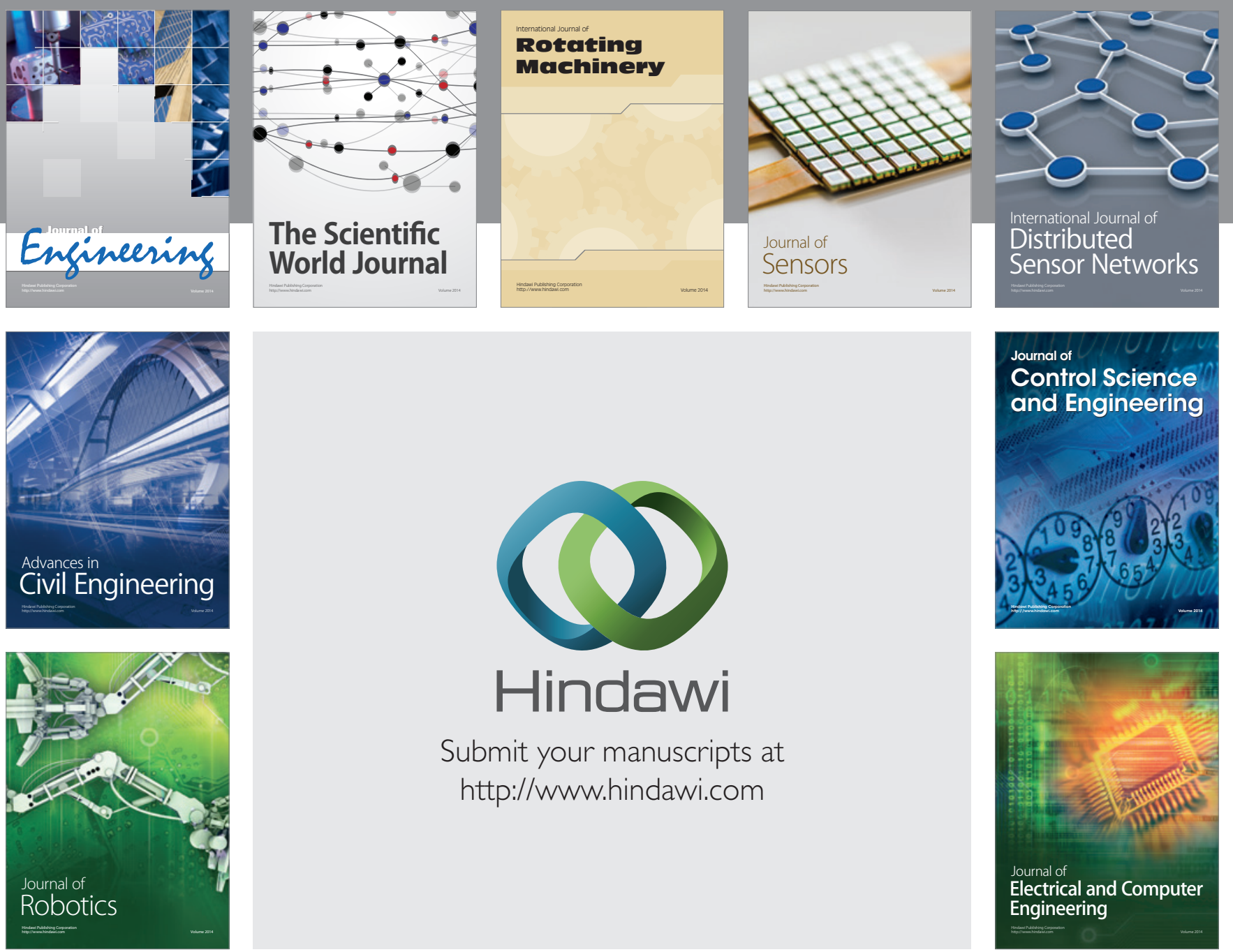

Submit your manuscripts at

http://www.hindawi.com
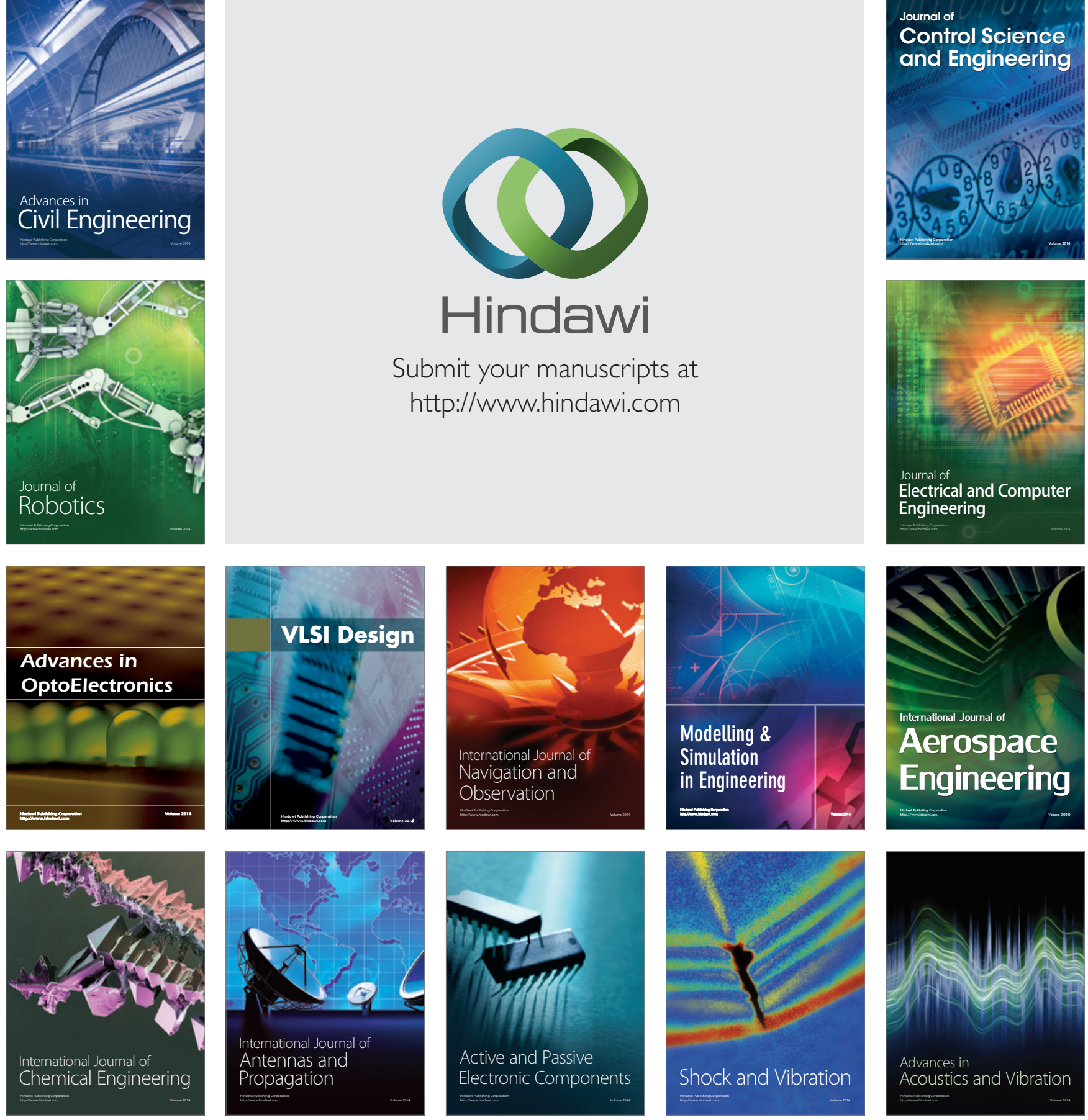